\title{
Genomic and clinical characteristics of six patients with partially overlapping interstitial deletions at $10 \mathrm{p} 12 \mathrm{p} 11$
}

\author{
Christian Wentzel $^{1}$, Evica Rajcan-Separovic ${ }^{2}$, Claudia AL Ruivenkamp ${ }^{3}$, Sandra Chantot-Bastaraud ${ }^{4}$, \\ Corinne Metay ${ }^{5}$, Joris Andrieux ${ }^{6}$, Göran Annerén ${ }^{1}$, Antoinet CJ Gijsbers ${ }^{3}$, Luc Druart ${ }^{4}$, Capucine Hyon ${ }^{7}$, \\ Marie-France Portnoi ${ }^{4}$, Eva-Lena Stattin ${ }^{8}$, Catherine Vincent-Delorme ${ }^{6}$, Sarina G Kant ${ }^{3}$, Michelle Steinraths ${ }^{9}$, \\ Sandrine Marlin ${ }^{4}$, Irina Giurgea ${ }^{5}$ and Ann-Charlotte Thuresson ${ }^{\star, 1}$
}

With the clinical implementation of genomic microarrays, the detection of cryptic unbalanced rearrangements in patients with syndromic developmental delay has improved considerably. Here we report the molecular karyotyping and phenotypic description of six new unrelated patients with partially overlapping microdeletions at $10 \mathrm{p} 12.31 \mathrm{p} 11.21 \mathrm{ranging}$ from 1.0 to $10.6 \mathrm{Mb}$. The smallest region of overlap is $306 \mathrm{~kb}$, which includes WAC gene, known to be associated with microtubule function and to have a role in cell division. Another patient has previously been described with a $10 \mathrm{Mb}$ deletion, partially overlapping with our six patients. All seven patients have developmental delay and a majority of the patients have abnormal behaviour and dysmorphic features, including bulbous nasal tip, deep set eyes, synophrys/thick eyebrows and full cheeks, whereas other features varied. All patients also displayed various visual impairments and six out of seven patients had cardiac malformations. Taken together with the previously reported patient, our study suggests that the detected deletions may represent a new contiguous gene syndrome caused by dosage-sensitive genes that predispose to developmental delay.

European Journal of Human Genetics (2011) 19, 959-964; doi:10.1038/ejhg.2011.71; published online 27 April 2011

Keywords: $10 p$ deletion; developmental delay; dysmorphic features; learning disability; mental retardation; WAC

\section{INTRODUCTION}

Today, genome-wide microarray-based screening for the detection of copy number variations $(\mathrm{CNV})$ is routinely offered to patients with developmental delay (DD) and dysmorphic features. The outcome of this is an increasing number of reported newly detected syndromes, and databases like DECIPHER (http://decipher.sanger.ac.uk/) and Ecaruca (http://agserver01.azn.nl:8080/ecaruca/ecaruca.jsp) have become important tools in collecting clinical information for detected chromosomal imbalances.

The recurrent CNVs associated with new syndromes are in some cases fairly consistent in size with reproducible breakpoints that map to areas of low-copy repeats, as for example in Smith Magenis and Williams-Beuren syndrome. ${ }^{1}$ On the other hand, other genomic abnormalities, although mapping to the same chromosomal region, may show a range of sizes and variable breakpoints with small areas of overlap like the $2 \mathrm{q} 33.1^{2}$ and $12 \mathrm{q} 14$ microdeletion syndromes., ${ }^{3,4}$

Recurrent chromosomal abnormalities involving the short arm of chromosome 10 have been rarely reported, and involve for example the DiDeorge syndrome region 2 (MIM 601362), with breakpoints mapping at 10p14-p13. Here we report six patients presenting with partially overlapping microdeletions of a more proximal region of the short arm of chromosome 10 (10p12.31p11.21), ranging from 1 to $10 \mathrm{Mb}$, with a common overlap of $306 \mathrm{~kb}$. There is one previous report of a patient with DD and dysmorphic features presenting with a microdeletion within this region detected with arrayCGH. ${ }^{5}$ The seven patients share some phenotypic traits and dysmorphic features. We describe the phenotypic traits that are shared between all individuals and suggest that microdeletions of 10p12.31p11.21 might be associated with a new clinically recognisable microdeletion syndrome.

\section{MATERIALS AND METHODS \\ Patients \\ DNA studies were performed after informed consent was obtained for all patients. Genomic DNA was extracted from peripheral blood leukocytes from patients using standard procedures. All patients showed a normal chromosomal karyotype by G-banding, except patients 4 and 5 who had a visible interstitial $10 \mathrm{p}$ deletion.}

\section{Microarray analysis}

The deletion in patient 1 was detected by an Affymetrix Genome-Wide SNP Array 6.0 (Affymetrix Inc., Santa Clara, CA, USA). Data analysis was carried out with Genotyping Console 4.0.

\footnotetext{
${ }^{1}$ Department of Immunology, Genetics and Pathology, The Rudbeck Laboratory, Uppsala University, Uppsala, Sweden; ${ }^{2}$ The Child and Family Research Institute and University of British Columbia, Vancouver, British Columbia, Canada; ${ }^{3}$ Department of Clinical Genetics, Center for Human and Clinical Genetics, Leiden University Medical Center (LUMC), Leiden, The Netherlands; ${ }^{4}$ Service de Génétique, Hôpital Armand Trousseau, Paris, France; ${ }^{5}$ Service de Biochimie-Génétique, Plateforme de Génomique Constitutionnelle, Hôpital Henri Mondor, Créteil, France; ${ }^{6}$ Laboratoire de Génétique Médicale, Hopital Jeanne de Flandre, CHRU de Lille, France; ${ }^{7}$ Universite Paris decarte, Service de génétique hôpital necker Paris, Paris, France; ${ }^{8}$ Department of Medical Biosciences, Medical and Clinical Genetics, Umeå University, Umeå, Sweden; ${ }^{9}$ Department of Laboratory Medicine, Pathology and Medical Genetics, Victoria General Hospital, Victoria, British Columbia, Canada

*Correspondence: Dr A-C Thuresson, Department of Immunology, Genetics and Pathology, The Rudbeck Laboratory, Uppsala University, Uppsala 751 85, Sweden.

Tel: +46 18611 2441; Fax: +46 18 554025; E-mail: ann-charlotte.thuresson@igp.uu.se

Received 20 December 2010; revised 8 March 2011; accepted 9 March 2011; published online 27 April 2011
} 
Agilent array was used to detect deletions in patients 2, 4, 5 and 6 . In patients 2 and 5, the deletion was detected by Agilent Human 105A array and in patient 6 with Agilent Human 244K array (Agilent, Santa Clara, CA, USA). Data analysis was carried out with DNA Analytics 4.0.76. In patient 4, the deletion was mapped using the Agilent Human 44K array. An Agilent Human $105 \mathrm{~A}$ array was later used to refine the breakpoints of the detected deletion. Confirmation of the deletion and parental analysis of patient 5 was carried out by using Cytochip v2.0 microarray (Bluegnome, Cambridge, UK) and data analysis with BlueFuse for Microarrays v.3.5 (Bluegnome, Cambridge, UK).

In patient 3, Illumina's HumanHap300 Genotyping BeadChip was used to detect CNVs. Data analysis was carried out with BeadStudio version 3.0. Parental analysis of patient 3 was performed with the same method.

All microarray and data analysis were performed in accordance with the manufacturer's instructions.

\section{MLPA}

Confirmation of detected CNVs of patients 1 and 3, and parental analysis of patient 1 , was carried out by using synthetic MLPA probes specifically designed to cover the region of interest, as previously described. ${ }^{6,7}$ Data analysis was performed with the GeneMarker software 1.85 (Softgenetics, State College, PA, USA).

\section{FISH}

Confirmation of microarray data and parental analysis of patients 2, 4 and 6 was performed by FISH analysis using BAC probes within the deleted region following standard techniques. ${ }^{8}$

\section{CASE REPORT}

Clinical features of all six patients are described in detail below and summarised in Table 1 .

\section{Patient 1}

This 6-year-old female patient (Figure 1a) was the first child born to non-consanguineous, healthy parents, both of 31 years of age at the time of birth. The family history was not contributory. The patient was delivered by scheduled caesarean section at $38+6$ weeks after an uneventful pregnancy. Apgar scores were 8-7-8. Birth weight was $2940 \mathrm{~g}$ ( $-1.5 \mathrm{SD} ; 20$ th percentile), length $49 \mathrm{~cm}$ ( $-0.5 \mathrm{SD} ; 45 \mathrm{th}$ percentile) and head circumference (HC) $35 \mathrm{~cm} \mathrm{(+1.4SD;} \mathrm{75th}$ percentile). She was immediately transferred to the neonatology ward after birth due to hypothermia, hypoglycaemia and respiratory problems. The rest of the neonatal period was without complications.

At the age of 9 months, concerns regarding DD and hypotonia were noted. She started walking at the age of 1.5 years. She spoke her first words when she was $2.5-3$ years old. Her speech has improved, but is still somewhat limited compared with other children of her age. At 6 years of age, she was no longer hypotonic but had problems with spasticity in her calves, especially on her left calf. Review of systems shows hyperopia, strabismus, episodic constipation and celiac disease. Hearing is normal. In terms of behavioural phenotype, she is hyperactive, gets easily frustrated and has aggressive outbursts. She does not show any stereotypic or self-mutilating behaviour. Investigations include an echocardiogram showing a ductus arteriosus that did not require treatment. MRI of the brain performed at 4 months of age and 2.5 years were normal. Because of her short stature, a screening for TSH, GH and IGF1 hormone levels was performed, which were all normal. Clinical examination at 4 and 6 years of age showed the following dysmorphic features: frontal bossing, short palpebral fissures, deep set eyes, low set eyebrows, synophrys, flat nose with a bulbous nasal tip, low posterior hairline, low set nipples and hirsutism, especially on the legs.

\section{Patient 2}

This 5-year-old female patient (Figure 1b) was the second child born to non-consanguineous, healthy parents. The patient was delivered by caesarean section at full term, Apgar scores were 8-9-9. Birth weight was $3645 \mathrm{~g}(+0.6 \mathrm{SD}$; 60 th percentile), length $52 \mathrm{~cm}(+1.3 \mathrm{SD}$; 80th percentile) and $\mathrm{HC} 37 \mathrm{~cm}$ (+3SD; 99th percentile). The mother was 36 and the father was 45 years old at the time of birth. During the neonatal period, she had feeding problems and problems with constipation.

From an early age, she has had pronounced sleep disturbance with very little sleep apparently required. She is currently on melatonin and clonidine, and without medication, she is reported to not sleep at all for several nights. She has moderate DD including walking and first words at age 2 years, as well as only starting smiling and being socially engaging around the same time. She is extremely hyperactive but quite clumsy, with a wide-based gait and dragging her left leg behind when walking. She has some autistic traits including a history of repetitive behaviours such as rocking and narrow interests. She interacts with her parents but does not play well with other children. She has problems with pronunciation of most words and an unusual voice. She has astigmatism with decreased vision in her left eye. Hearing was normal when tested at age 2. MRI of the brain showed a small left frontal intraventricular lesion felt likely to be a cyst and normal ventricular size. Echocardiogram was normal.

Clinical examination at 2.5 and 4 years of age showed the following dysmorphic features: a low anterior hairline with a prominent supraorbital ridge, downslanting palpebral fissures, synophrys, short nose with a bulbous nasal tip, prominent premaxilla, short neck, inverted nipples, wide chest, hirsutism with prominent dark hair over her back, tapered digits with mildly hypoplastic nails on the fifth finger, and puffy dorsum of hands and feet. On examination at age 4 years, her height was $109 \mathrm{~cm}$ (+1.9SD; 90th percentile), weight $20 \mathrm{~kg}$ (+1.8 SD; 85th percentile) and HC $52 \mathrm{~cm}(+1.7 \mathrm{SD}$; 80th percentile).

\section{Patient 3}

This female patient (Figure 1c) was the second child born to nonconsanguineous, healthy parents. The mother and father were 24 and 26 years old, respectively, at the time of birth. The pregnancy was uneventful. Birth was at 38 weeks by a caesarean section because of transverse position. Birth weight was $2845 \mathrm{~g}(-1.5 \mathrm{SD}$; 15th percentile). Moderate DD was noted in the first year. She walked independently at 21 months. She also had speech delay and was diagnosed with autism spectrum disorder. Hearing is normal. Her vision is impaired because of amblyopia, astigmatism and moderate myopia. She had surgery for strabismus and an open ductus arteriosus. CT and MRI of the brain both showed no abnormalities.

At the age of 11 years, her height was $142 \mathrm{~cm}(-1.3 \mathrm{SD} ; 35 \mathrm{th}$ percentile), weight was $41.3 \mathrm{~kg}(+0.8 \mathrm{SD}$; 65 th percentile) and $\mathrm{HC}$ was $52.8 \mathrm{~cm}(-0.4 \mathrm{SD} ; 40$ th percentile). She was brachycephalic. She had small ears with a narrow ear canal and a slightly dysplastic helix, a broad nose with a bulbous nasal tip, abnormal palmar creases, a short fifth metacarpal on both sides and scoliosis. Her gait was stiff and she had normal tone.

\section{Patient 4}

This 3-year-old male patient (Figure 1d) was the first child born to non-consanguineous, healthy parents. At time of birth, the mother was 25 years old and the father 27 years. The patient was delivered by spontaneous delivery at 40 weeks. Birth weight was $2840 \mathrm{~g}(-1.5 \mathrm{SD}$; 15th percentile), length $47 \mathrm{~cm}(-1.5 \mathrm{SD} ; 15$ th percentile) and $\mathrm{HC}$ $34 \mathrm{~cm}(+0.5 \mathrm{SD} ; 60$ th percentile). He had aortic stenosis for which he had a surgery at 4 days of age.

He has gross motor delays including walking at 20 months. There is no known speech delay. He has hyperactivity requiring medical 


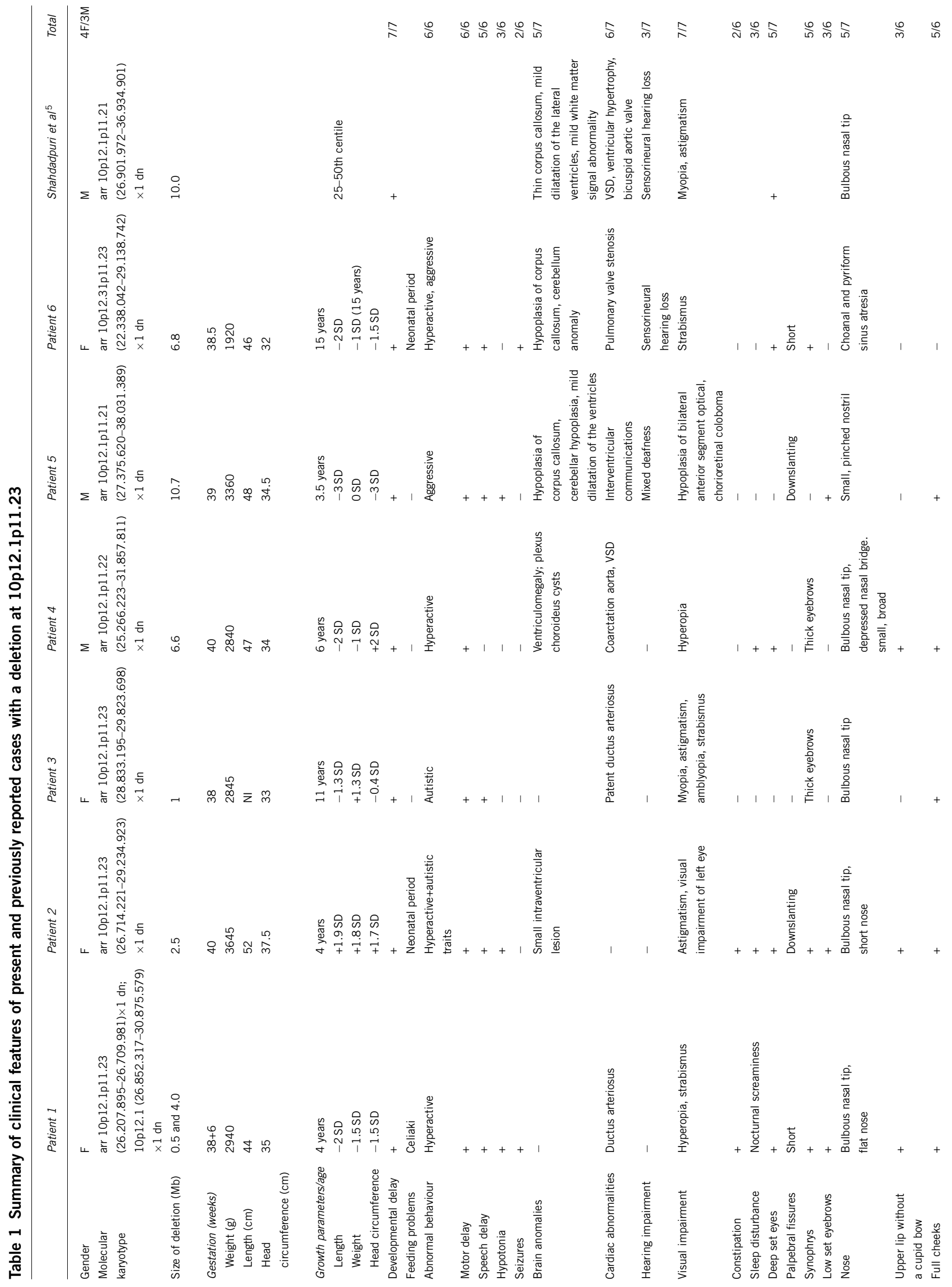



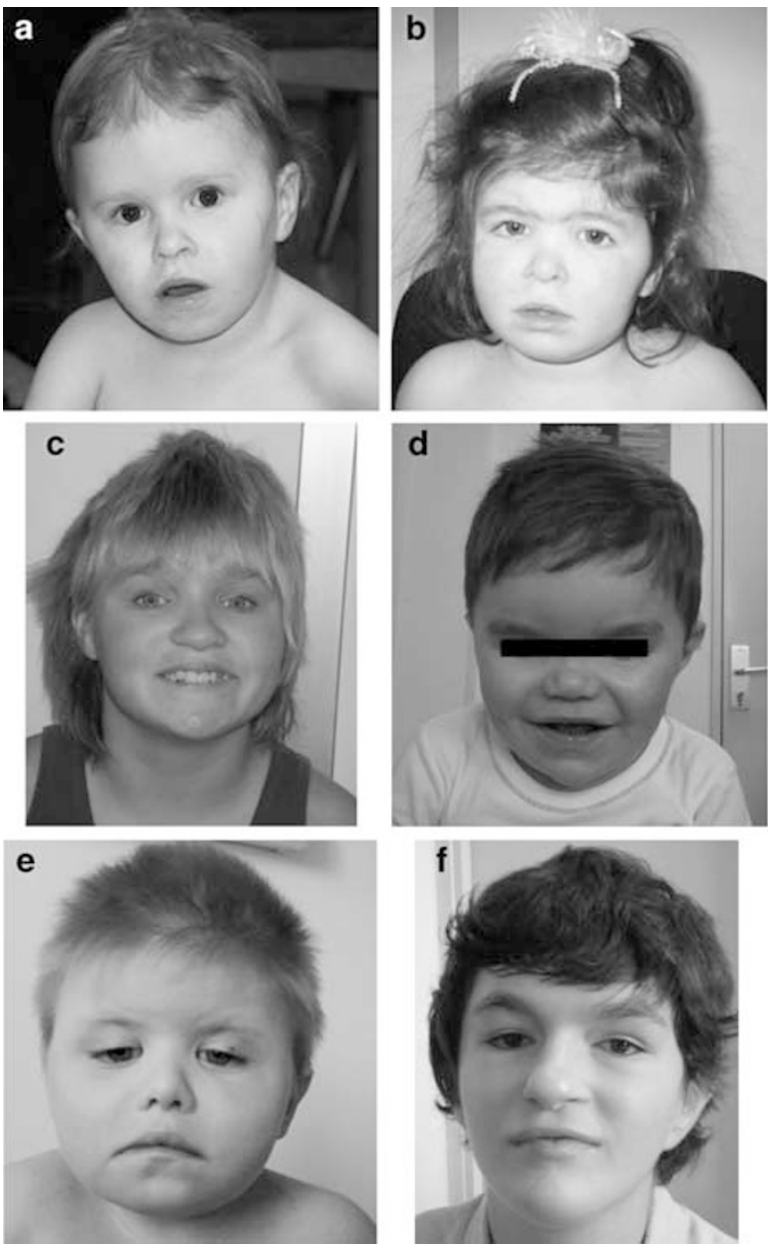

Figure 1 (a) Patient 1 at age 4, (b) patient 2 at age 2 years, 5 months, (c) patient 3 at 11 years of age, (d) patient 4 at 3 years, 9 months, (e) Patient 5 at 3 years, 9 months and (f) patient 6 at 14 years of age.

treatment. Review of systems shows lacrimal duct stenosis, hyperopia, bilateral inguinal hernia and unilateral cryptorchidism. X-rays showed a supernumerary rib. Moderate ventriculomegaly was noted on head ultrasound. His physical findings were worth noting for a depressed nasal bridge, thick eyebrows, macrocephaly and small, thick fingers.

\section{Patient 5}

This 4-year-old male patient (Figure 1e) was the second child born to non-consanguineous, healthy parents. The age of the mother and father at time of birth was 34 and 38 years old, respectively. At 35 weeks of gestation, cerebral ventriculomegaly and ventricular septal defect (VSD) were detected by foetal ultrasonography. Antenatal karyotype was undertaken and an interstitial 10p deletion was diagnosed, with normal FISH for DGCR2 locus. The patient was born at 39 weeks of gestation with normal growth parameters: weight was $3360 \mathrm{~g}(+0.5 \mathrm{SD}$; 55 th percentile), length $48 \mathrm{~cm}(-1 \mathrm{SD} ; 25$ th percentile) and HC $34.5 \mathrm{~cm}(+0.8 \mathrm{SD}$; 65th percentile). During the neonatal period, he required 1 month of oxygen therapy for pulmonary arterial hypertension. He had multiple VSDs (a perimembranous defect partially obstructed by aneurysm of the membranous septum and two small trabecular defects), which closed spontaneously within the first year. He had delayed psychomotor development, including not sitting until 3 years. At age 4 years, he was not walking without 
support and only had two or three single words. He had episodes of aggressive behaviour. Ophthalmic examination revealed chorioretinal coloboma of the right eye. Severe bilateral sensorineural and conductive hearing loss was diagnosed at 1 year of age. Transtympanic drains in the treatment of serous otitis improved the hearing loss. CT scan of the temporal bone was normal. He also had bilateral cryptorchidism requiring surgery at 20 months of age. Brain MRI showed ventriculomegaly, cerebellar hypoplasia and bilateral anterior optic nerve hypoplasia.

On physical examination, he had a normal weight for his age, but developed a postnatal growth retardation $(-3 \mathrm{SD})$ and microcephaly $(-4 \mathrm{SD})$. He had axial hypotonia and peripheral hypertonia. Facial dysmorphia included bilateral ptosis and epicanthal folds, anteverted nares, short neck, dysplastic ears, a large mouth with Cupid's bow upper lip, downturned corners and bifid tongue. Camptodactyly and syndactyly of the toes were noted.

\section{Patient 6}

This girl (Figure 1f) is the only child of parents with no medical concerns. She was born at 38.5 weeks of gestation with growth retardation: weight $1920 \mathrm{~g}$ ( $-3 \mathrm{SD}$; <3rd percentile), length $46 \mathrm{~cm}$ ( $-2 \mathrm{SD}$; 3rd percentile) and HC $32 \mathrm{~cm}(-1.5 \mathrm{SD} ; 15$ th percentile). The age of the mother and father at time of birth was 36 and 39 years old, respectively. Congenital anomalies include right choanal atresia, stenosis of the left pyriform sinus and pulmonary valve stenosis. She showed significant difficulty in swallowing. Because of speech delay, a hearing test was performed at 3 years of age and bilateral sensorineural hearing loss was diagnosed. The hearing loss was progressive, and at 14 years of age, she had a profound deafness. She has moderate DD including walking at age 3 . She has attention-deficit hyperactivity disorder treated with methylphenidate and occasionally has selfaggressive behaviour. A cerebral MRI performed at age 7 years due to seizures showed hypoplasia of the corpus callosum and cerebellar abnormalities. Her menstrual periods started at the age of 8 years and remain irregular.

At the time of clinical examination at the age of 14 years, her weight, height and $\mathrm{HC}$ are normal. She has facial asymmetry, dysplastic ears, enophthalmos, synophrys, arched eyebrows and slight inferior prognathism. The rest of her clinical examination is unremarkable.

\section{RESULTS}

Molecular results of the six patients are summarised in Figure 2. All deletions were de novo. The position of the array targets are according to NCBI36 (hg18).

In patient 1 , analysis by SNP array revealed two tandem deletions of 0.51 and $4.0 \mathrm{Mb}$, respectively, located at $10 \mathrm{p} 12.1$ and $10 \mathrm{p} 12.1 \mathrm{p} 11.23$ (26.207.895-26.709.981 and 26.852.317-30.875.579). The deletion could not be detected by MLPA in her parents.

In patient 2 , a $2.5 \mathrm{Mb}$ interstitial deletion was detected by an oligonucleotide microarray at $10 \mathrm{p} 12.1 \mathrm{p} 11.23$ (26.714.22129.234.923). Parental investigations by FISH analysis proved the deletion to be de novo.

In patient 3 , a $1 \mathrm{Mb}$ deletion was detected at 10p11.23 (28.833.19529.823.698) with an SNP array. The deletion could not be detected by the same method in her parents. FISH analysis of the mother revealed no structural abnormalities of chromosome 10 .

In patient 4 , microarray analysis revealed a $6.6 \mathrm{Mb}$ deletion (25.266.223-31.857.811) located at 10p12.1p11.22. The deletion could not be detected in the parents by FISH analysis.

In patient 5, microarray analysis revealed a $10.7 \mathrm{Mb}$ deletion at 10p12.1p11.21 (27.375.620-38.031.389). No deletion could be detected in his parents by BAC arrayCGH.

In patient 6 , a $6.8 \mathrm{Mb}$ interstitial deletion was detected at 10p12.31p11.23 (22.338.042-29.138.742) by an oligonucleotide microarray. Parental investigations by FISH proved the deletion to be de novo.

\section{DISCUSSION}

Here we present six new patients with deletions of different sizes encompassing $10 \mathrm{p} 12.31 \mathrm{p} 11.21$. None of the patients share common breakpoints. Non-homologous end joining (NHEJ) or FoSTeS (fork stalling and template switching) might be likely mechanisms for recurrence of deletions in this region of chromosome 10 as the breakpoints do not contain any segmental duplications required for NAHR. ${ }^{1,9}$

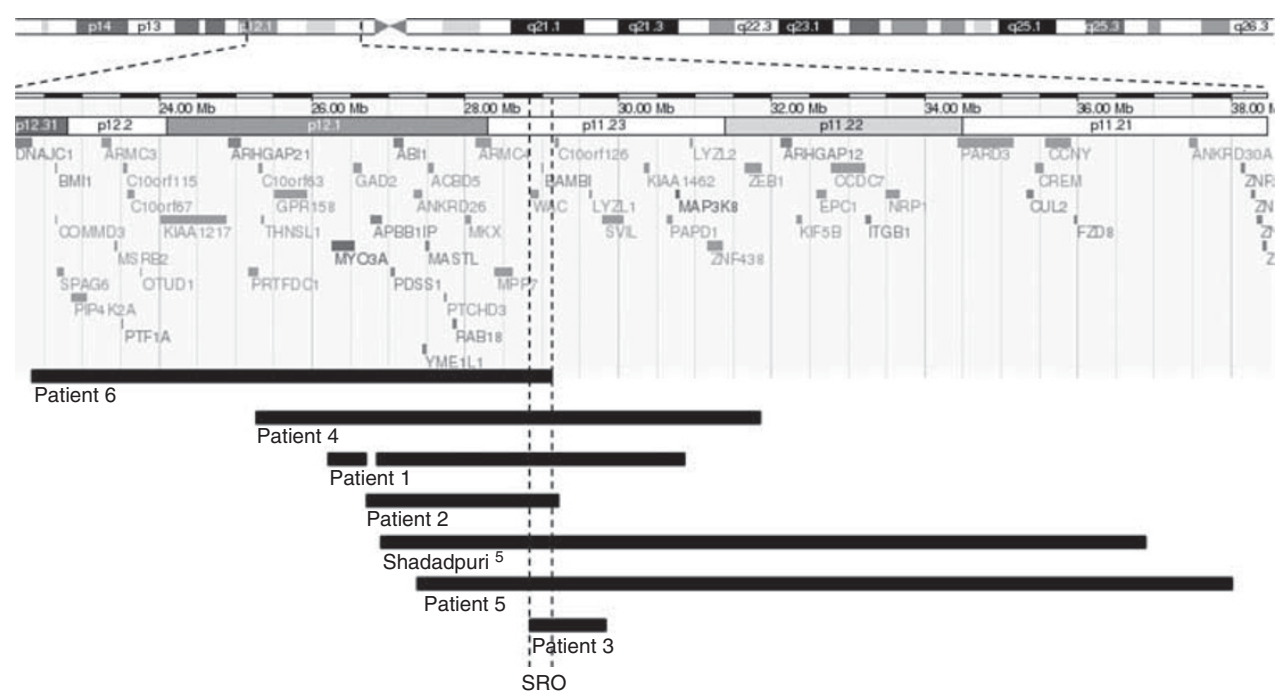

Figure 2 Schematic illustration of chromosome 10 displaying the deletions at $10 \mathrm{p} 12 \mathrm{p} 11$ in patients $1-6$ and patient by Shadadpuri et a/ ${ }^{5}$ detected by microarray using the Ensembl browser. Deleted regions for the respective cases are shown as black bars. Smallest region of overlap (SRO) is indicated by vertical dotted lines. Positions are according to NCBI36 (hg18). 
Comparison of clinical features of our six patients showed that they share some similarities such as DD (6/6), motor (6/6) and speech delay (5/6), hyperactivity (4/6) and hypotonia (3/6). They also display dysmorphic features such as: synophrys/thick eyebrows (5/6), short neck (3/6), deep set eyes (4/6), low set eyebrows (3/6), bulbous nose $(4 / 6)$ and full cheeks (5/6). All patients also had different kinds of visual impairments. Our six patients share some features with the previously described patient with a 10 p $12-$ p 11 deletion. ${ }^{5}$ Clinical data for this patient are summarised in Table 1 (genomic data kindly provided by Dr Nicole de Leeuw chr10: 26.901.972-36.934.901 (hg18)). The similarities between our six patients and the patient reported in the literature included DD and a majority of the patients have abnormal behaviour and dysmorphic features, including bulbous nasal tip, deep set eyes, synophrys/thick eyebrows and full cheeks, whereas other features varied. In contrast, the previously described patient had unique changes detected on X-ray. Plain radiography showed pseudoarthrosis of the right clavicle and a significant beaten copper appearance of the cranium without any evidence of sutural synostosis. None of our patients have had X-rays to assess for these abnormalities.

Cardiac abnormalities, mostly conotrucal heart defects, were present in patients 1 and 3-6, as well as in the previously published patient. All the patients, except patient 6 , shared a $589 \mathrm{~kb}$ deletion (chr10: 29.823.698-29.234.923 (hg18)). Two protein-coding genes are present in the region: LYZL1 and SVIL. SVIL is suggested to have a role as a high-affinity link between the actin cytoskeleton and the membrane and appears to be involved in myosin II assembly. In addition, two non-coding RNAs, mir-604 and mir-938, are located within this commonly deleted region. Several reports have indicated a role for non-coding RNAs in the molecular pathogenesis of different developmental disorders. ${ }^{10}$ However, the role they might have in the development of the heart is still unknown.

The smallest region of overlap in all seven patients is $306 \mathrm{~kb}$ in size and contains only two genes, BAMBI and WAC (Figure 2). BAMBI encodes a transmembrane glycoprotein related to the type I receptors of the TGF- $\beta$ family, whose members have important roles in signal transduction in many developmental and pathological processes. The encoded protein, however, is a pseudoreceptor, lacking an intracellular serine/threonine kinase domain required for signalling, and was recently shown to be dispensable for mouse embryonic development and postnatal survival, ${ }^{11}$ but may have a role in pain modulation. ${ }^{12}$ The protein encoded by WAC represents a subunit of the Augumin complex, which is required for centrosome-independent microtubule generation within the spindle. Wac depletion in cultured Drosophila S2 cells has been shown to cause severe defects in spindle assembly. ${ }^{13,14}$ In developing Drosophila, WAC is required for cell proliferation, but its homozygous or heterozygous deletions were not found to be lethal. Instead, in somatic cells, the deletion of WAC resulted in delayed mitotic progression with a proper chromosome segregation in general. The defects of genes coding for proteins responsible for microtubule function has previously been associated with neurodevelopmental disorders with dysmorphic features, ${ }^{15}$ with MAPT representing one recent example. This gene is considered to be the candidate for the features of the recurrent $17 \mathrm{q} 21$ deletion syndrome, ${ }^{16}$ which interestingly shows DD, facial dysmorphisms including a long face, a tubular or pear-shaped nose and a bulbous nasal tip, as well as eye abnormalities among the recurrent features. It is therefore possible that WAC is one of the candidates for the DD, which is the only feature common in all seven patients. However, functional studies are required to draw any conclusions if WAC could be a candidate gene responsible for the DD.

In conclusion, we report six new unrelated patients with a deletion at $10 \mathrm{p} 12 \mathrm{p} 11$ that together with a previously published case share a similar clinical phenotype characterised by DD, abnormal behaviour, visual impairment, cardiac abnormalities and dysmorphic features like deep set eyes, synophrys/thick eyebrows, full cheeks and a bulbous nasal tip.

The possible role of WAC gene as a candidate gene for DD was raised, but further molecular characterisation of additional patients is needed to further delineate its role in abnormal human development.

\section{CONFLICT OF INTEREST}

The authors declare no conflict of interest.

\section{ACKNOWLEDGEMENTS}

We thank all of the families for agreeing to participate in this study. This work was supported by grants from the Sävstaholm Society, the Borgström Foundation, Foundation Jerome Lejeune and the Canadian Institutes of Health Research (ERS). ERS acknowledges the Michael Smith Foundation of Health Research Scholar Award. We are grateful for technical assistance and scientific discussion to Laure Lecerf, Audrey Briand and Sandra Whalen.

1 Stankiewicz P, Lupski JR: Structural variation in the human genome and its role in disease. Annu Rev Med 2010; 61: 437-455.

2 Van Buggenhout G, Van Ravenswaaij-Arts C, Mc Maas N et al: The del(2)(q32.2q33) deletion syndrome defined by clinical and molecular characterization of four patients. Eur J Med Genet 2005; 48: 276-289.

3 Lynch SA, Foulds N, Thuresson AC et al: The 12q14 microdeletion syndrome: six new cases confirming the role of HMGA2 in growth. Eur J Hum Genet 2011; e-pub ahead of print 26 January 2011.

4 Menten B, Buysse K, Zahir F et al: Osteopoikilosis, short stature and mental retardation as key features of a new microdeletion syndrome on 12q14. J Med Genet 2007; 44: 264-268.

5 Shahdadpuri R, de Vries B, Pfundt R, de Leeuw N, Reardon W: Pseudoarthrosis of the clavicle and copper beaten skull associated with chromosome 10p11.21p12.1 microdeletion. Am J Med Genet A 2008; 146A: 233-237.

6 Stern RF, Roberts RG, Mann K, Yau SC, Berg J, Ogilvie CM: Multiplex ligationdependent probe amplification using a completely synthetic probe set. Biotechniques 2004; 37: 399-405.

7 Thuresson AC, Bondeson ML, Edeby $\mathrm{C}$ et al: Whole-genome array-CGH for detection of submicroscopic chromosomal imbalances in children with mental retardation. Cytogenet Genome Res 2007; 118: 1-7.

8 Dauwerse JG, Jumelet EA, Wessels JW et al: Extensive cross-homology between the long and the short arm of chromosome 16 may explain leukemic inversions and translocations. Blood 1992; 79: 1299-1304.

9 Zhang F, Gu W, Hurles ME, Lupski JR: Copy number variation in human health, disease, and evolution. Annu Rev Genomics Hum Genet 2009; 10: 451-481.

10 Szulwach KE, Jin P, Alisch RS: Noncoding RNAs in mental retardation. Clin Genet 2009; 75: 209-219.

11 Chen J, Bush JO, Ovitt CE, Lan Y, Jiang R: The TGF-beta pseudoreceptor gene Bambi is dispensable for mouse embryonic development and postnatal survival. Genesis 2007; 45: 482-486.

12 Tramullas M, Lantero A, Diaz A et al: BAMBI (bone morphogenetic protein and activin membrane-bound inhibitor) reveals the involvement of the transforming growth factor-beta family in pain modulation. J Neurosci 2010; 30: 1502-1511.

13 Goshima G, Mayer M, Zhang N, Stuurman N, Vale RD: Augmin: a protein complex required for centrosome-independent microtubule generation within the spindle. J Cell Biol 2008; 181: 421-429.

14 Meireles AM, Fisher KH, Colombie N, Wakefield JG, Ohkura H: Wac: a new Augmin subunit required for chromosome alignment but not for acentrosomal microtubule assembly in female meiosis. J Cell Biol 2009; 184: 777-784.

15 Jaglin XH, Chelly J: Tubulin-related cortical dysgeneses: microtubule dysfunction underlying neuronal migration defects. Trends Genet 2009; 25: 555-566.

16 Koolen DA, Vissers LE, Pfundt $R$ et al: A new chromosome 17q21.31 microdeletion syndrome associated with a common inversion polymorphism. Nat Genet 2006; 38 : 999-1001. 